\title{
Circulation-dominated solar shell dynamo models with positive alpha-effect
}

\author{
M. Küker^, G. Rüdiger, and M. Schultz \\ Astrophysikalisches Institut Potsdam, An der Sternwarte 16, 14482 Potsdam, Germany \\ Received 7 November 2000 / Accepted 9 May 2001

\begin{abstract}
We present shell dynamo models for the solar convection zone with positive $\alpha$-effect in the northern hemisphere and a meridional circulation which is directed equatorward at the bottom and poleward at the top of the convection zone. Two different rotation patterns are used: a simple variation of the rotation rate with depth and the rotation law as derived by helioseismology. Depending on the Reynolds number associated with the meridional flow, the dynamo shows a whole "zoo" of solutions. For sufficiently small values of the eddy magnetic diffusivity $\left(10^{11} \mathrm{~cm}^{2} / \mathrm{s}\right)$, field advection by the meridional flow becomes dominant and even changes the character of the butterfly diagram. Flow amplitudes of a few $\mathrm{m} / \mathrm{s}$ are then sufficient to turn the originally "wrong" butterfly diagram into a "solar-type" butterfly diagram, i.e. with activity belts drifting equatorward. This effect can easily be demonstrated with a super-rotation law $(\partial \Omega / \partial r>0)$ with $\Omega$ independent of the latitude. The situation is much more complicated for the "real" rotation law with the observed strong negative shear at high latitudes. With zero meridional flow, oscillating solutions are found without any latitudinal migration of the toroidal field belts, neither poleward nor equatorward. Small but finite flow amplitudes cause the magnetic field belts to drift poleward while in case of fast flow they move equatorward.
\end{abstract}

Key words. magnetohydrodynamics - Sun: interior - Sun: magnetic field

\section{Introduction}

Our understanding of stellar activity is based on dynamo theory, which tells us that magnetic activity strongly depends on the star's internal rotation law. Dynamos in differentially rotating stars differ from those in stars that rotate rigidly because rotational shear generates a strong toroidal field and enforces an axisymmetric field geometry. The only star with known internal rotation is the Sun. Helioseismology gives us detailed information about the internal rotation profiles apart from the well-known surface phenomenon of the equatorial acceleration of $\delta \Omega \simeq$ 0.06 per day. One finds super-rotation beneath the equator and sub-rotation beneath the poles. Close to the equatorial plane, the rotation rate is essentially constant on cylindrical surfaces, while close to the poles the surfaces of isorotation are rather disk-shaped.

Not only the differential rotation but also the meridional flow (see Fig. 1) will influence the mean-field dynamo (Roberts \& Stix 1972). This influence can be expected to be just a modification if its characteristic time-scale $\tau_{\text {drift }}$

Send offprint requests to: M. Küker, e-mail: kueker@astro.uni-jena.de

* Present address: Astrophysikalisches Institut und Universitätssternwarte, Schillergässchen 2-3, 07745 Jena, Germany.

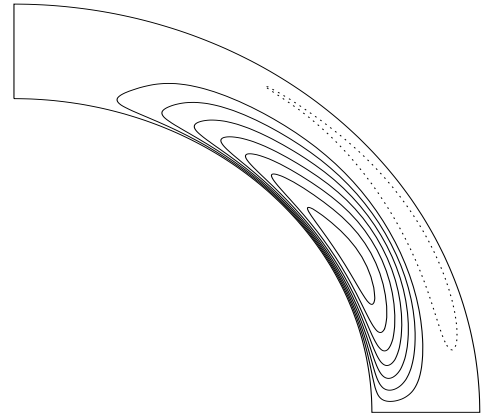

Fig. 1. The meridional flow pattern after the theory of differential rotation by Kitchatinov \& Rüdiger (1999). In the present dynamo calculations the weak upper cell has been ignored. Note the basic drift concentrated to the bottom of the convection zone where it flows equatorward.

exceeds the (half-)cycle time $\tau_{\text {cyc }}$ of about 11 yr. With $\tau_{\text {drift }} \simeq R / u_{\text {drift }}$ we find $u_{\text {drift }} \simeq 2 \mathrm{~m} / \mathrm{s}$ as a critical value for the flow velocity. If the flow is faster, as predicted by the most recent theories of solar rotation (cf. Fig. 2), the modification might be drastic.

If, in particular, field advection by the flow counteracts the propagation of the dynamo wave, i.e. if the drift is poleward at the bottom of the convection zone, 

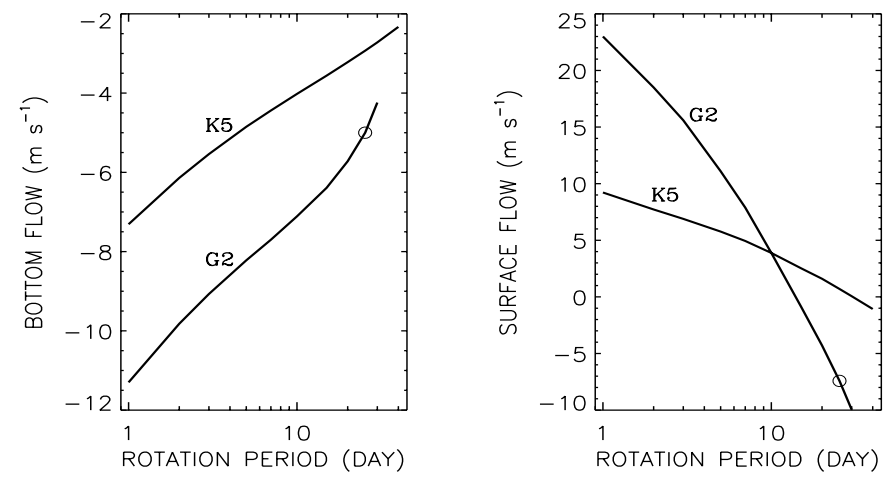

Fig. 2. The meridional circulation at mid-latitudes $\left(45^{\circ}\right)$ at the bottom (Left) and the top (Right) of the convection zone for both a solar model and a K5 main-sequence star as a function of rotation period (Kitchatinov \& Rüdiger 1999). The circle represents the solar case. The flow at the bottom is equatorward (as it is the flow at the surface, there are 2 cells in radius).

the dynamo might fail to maintain a solar-type magnetic cycle. On the other hand, an equatorward directed meridional flow can produce the observed solar-type butterfly diagram even in the case that a circulation-free dynamo would produce an antisolar-type butterfly diagram (Choudhuri et al. 1995; Dikpati \& Charbonneau 1999). The overall behavior of such a dynamo with super-rotation at the bottom of the convection zone (and a perfectly conducting radiative core) is demonstrated in the present paper. Particular attention is focused on the phase relation between the toroidal and the radial field components, which is negative in the solar photosphere (Stix 1973). It is almost impossible to explain this observation by virtue of an $\alpha \Omega$-dynamo and a rotation law with positive shear (Schlichenmaier \& Stix 1995). We shall see that this situation is changed if meridional circulation is taken into account.

\section{The dynamo model}

In the following, the dynamo equations are given with the inclusion of the meridional circulation. For axisymmetry the mean flow in spherical polar coordinates is given by

$\boldsymbol{u}=\left(u_{\mathrm{r}}, u_{\theta}, r \sin \theta \Omega\right)$.

With a similar notation the magnetic field with $\operatorname{div} \boldsymbol{B}=0$ is

$\boldsymbol{B}=\left(\frac{1}{r^{2} \sin \theta} \frac{\partial A}{\partial \theta},-\frac{1}{r \sin \theta} \frac{\partial A}{\partial r}, B\right)$,

with $A$ as the poloidal-field potential and $B$ as the toroidal field. Their evolution can be described by the two equations

$$
\begin{aligned}
\frac{\partial A}{\partial t} & +(\boldsymbol{u} \cdot \nabla) A=\alpha s B+\sqrt{\eta_{\mathrm{T}}} \frac{\partial}{\partial r}\left(\sqrt{\eta_{\mathrm{T}}} \frac{\partial A}{\partial r}\right) \\
& +\eta_{\mathrm{T}} \frac{\sin \theta}{r^{2}} \frac{\partial}{\partial \theta}\left(\frac{1}{\sin \theta} \frac{\partial A}{\partial \theta}\right)
\end{aligned}
$$

$$
\begin{aligned}
\frac{\partial B}{\partial t} & +\rho s(\boldsymbol{u} \cdot \nabla) \frac{B}{s \rho}=\frac{1}{r}\left(\frac{\partial \Omega}{\partial r} \frac{\partial A}{\partial \theta}-\frac{\partial \Omega}{\partial \theta} \frac{\partial A}{\partial r}\right) \\
& -\frac{1}{s} \frac{\partial}{\partial r}\left(\alpha \frac{\partial A}{\partial r}\right)-\frac{1}{r^{3}} \frac{\partial}{\partial \theta}\left(\frac{\alpha}{\sin \theta} \frac{\partial A}{\partial \theta}\right) \\
& +\frac{1}{s} \frac{\partial}{\partial r}\left(\sqrt{\eta_{\mathrm{T}}} \frac{\partial\left(\sqrt{\eta_{\mathrm{T}}} s B\right)}{\partial r}\right) \\
& +\eta_{\mathrm{T}} \frac{1}{r^{2}} \frac{\partial}{\partial \theta}\left(\frac{1}{s} \frac{\partial(s B)}{\partial \theta}\right)
\end{aligned}
$$

with $s=r \sin \theta$ (see Rüdiger \& Arlt 2000). The unusual form of the eddy diffusivity term is used to include the turbulent diamagnetism, which occurs in case of spatially inhomogeneous turbulence intensity and is a strong effect for low- $\eta_{\mathrm{T}}$ dynamos. To get a smooth transition at the bottom of the convection zone, located at a fraction radius of 0.7 , the profile

$$
\eta_{\mathrm{T}}=\eta_{\mathrm{c}}+\frac{1}{2}\left(\eta_{\mathrm{t}}-\eta_{\mathrm{c}}\right)(1+\operatorname{erf}(40(x-0.7)))
$$

is chosen, where $x=r / R_{\odot}$ is the fractional radius, erf denotes the error function, $\eta_{\mathrm{t}}$ is the eddy diffusivity, and $\eta_{\mathrm{c}}$ the magnetic diffusivity beneath the convection zone, usually chosen about two orders of magnitude smaller than $\eta_{\mathrm{t}}$. The factor of 40 in the argument of the error function defines the thickness of the transition region to be 0.05 solar radii.

The $\alpha$ effect is constrained to the upper half of the convection zone and low latitudes through a profile similar to that of Choudhuri et al. (1995),

$$
\begin{aligned}
\alpha= & \alpha_{0} q(\boldsymbol{B}) \cos \theta \sin ^{2} \theta \frac{1}{4}\left(1+\operatorname{erf}\left(40\left(x-x_{\mathrm{in}}\right)\right)\right) \\
& \times(1-\operatorname{erf}(40(x-1))),
\end{aligned}
$$

where $x_{\text {in }}=0.9$ and $q(\boldsymbol{B})$ is a quenching function which will be specified later. With a value of 0.75 instead of 0.9 for $x_{\text {in }}$ the $\alpha$ effect is present in the whole convection zone. The latter case is later referred to as a thick $\alpha$-layer. We assume a convection zone with constant density scale height, i.e.

$\rho=x^{-G}$

with $G=7$. For the meridional flow velocity the ansatz

$u_{\theta}=-\frac{u_{0} \psi}{\rho x} \frac{\mathrm{d} A_{2}}{\mathrm{~d} x} P_{2}^{1}$,

$u_{\mathrm{r}}=\frac{6 u_{0} \psi}{\rho x^{2}} A_{2} P_{2}$,

where the variation with latitude is determined by the functions

$P_{2}^{1}=3 \cos \theta \sin \theta$

$P_{2}=\frac{1}{2}\left(3 \cos ^{2} \theta-1\right)$,

and the dependence on radius by the function

$A_{2}=\sum_{n=0}^{7} \frac{G a_{n}+b_{n}}{G+c} x^{n}$ 


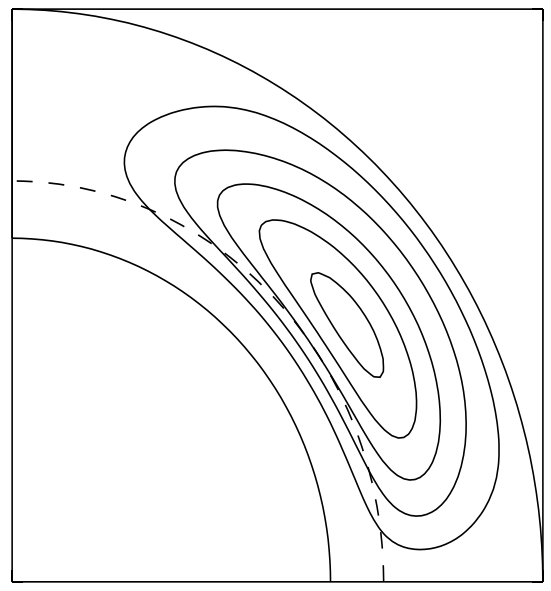

Fig. 3. Stream lines of the meridional flow in the northern hemisphere for $G=7$. The inner circle marks the lower boundary at $x=0.6$ while the dashed circle indicates the bottom of the convection zone at $x=0.7$. The circulation is clockwise for positive values of $u_{0}$.

Table 1. The coefficients of the expansion in Eq. (12).

\begin{tabular}{|c|c|c|}
\hline$n$ & $a_{n}$ & $b_{n}$ \\
\hline 0 & 1.0 & 35.79445814 \\
1 & -9.128928668 & -325.4816432 \\
2 & 35.39211526 & 1256.114922 \\
3 & -75.54955261 & -2667.188479 \\
4 & 95.93030242 & 3366.002169 \\
5 & -72.48891863 & -2525.564865 \\
6 & 30.19813615 & 1043.614976 \\
7 & -5.353153923 & -183.2915368 \\
\hline
\end{tabular}

then ensures that the boundaries are stress-free and mass conservation holds. The factor $\psi$ is determined by the condition:

$u_{0}=\max _{N}\left(u_{\theta}\right)$,

where the subscript $N$ indicates that the maximum ${ }^{1}$ refers to the northern hemisphere only. The coefficients given in Table 1 together with $c=35.7945$ yield $\psi=1.715 \times 10^{3}$ and a flow pattern that consists of one cell per hemisphere, as shown in Fig. 3. The direction of the flow depends on $u_{0}$. Positive values give clockwise while negative values result in anti-clockwise circulation.

The solar rotation law in the convection zone is modeled in two steps. In a first set of models we only adopt a super-rotation independent of latitude and concentrated at the bottom of the convection zone. Much more complicated is the application of the real solar rotation law derived by helioseismology. In order to produce a solartype butterfly diagram with $\boldsymbol{u}=0$ and with the observed super-rotation beneath the equator, the $\alpha$-effect must then

\footnotetext{
${ }^{1}$ More precisely, $u_{0}$ is the value of $u_{\theta}$ with the maximum absolute value.
}

be negative in the northern and positive in the southern hemisphere (Steenbeck \& Krause 1969; Parker 1987; Rüdiger \& Brandenburg 1995; see also the discussion by Moss \& Brooke 2000).

However, the positive values adopted in Fig. 4 (see Durney 1995, 1996) are much easier to understand than a negative $\alpha$-effect. Recent numerical simulations lead to the result that turbulence is much more magnetically driven than believed (cf. Brandenburg 1999). In particular the role of magnetic buoyancy appears quite important for the generation of $\alpha$-effect and angular momentum transport (cf. Brandenburg \& Schmitt 1998). So far, only the role of density stratification has been discussed for the $\alpha$ effect and its relation to kinetic helicity and anisotropy of the turbulence. Rüdiger et al. (2000) present an analysis of both effects, for a turbulence field driven by the Lorentz force in a non-stratified but rotating convection zone. Their main result confirms the numerical findings of Brandenburg \& Schmitt that the $\alpha$-effect is positive in the northern and negative in the southern hemisphere, just the opposite of what occurs for the current helicity, which is negative in the northern and positive in the southern hemisphere. A growing number of observations of the current helicity on the solar surface finds negative values in the northern and positive values in the southern hemisphere (Hale 1927; Seehafer 1990; Pevtsov et al. 1995; Abramenko et al. 1996; Bao \& Zhang 1998, see Low (1996) for a review).

We therefore assume that the $\alpha$-effect exists in the bulk of the convection zone, but not at its bottom. All the characteristic values for the $\alpha$-effect in this paper are attributed to its maximum within the convection zone. Throughout the whole paper we work with an $\alpha$-effect amplitude of order $1 \mathrm{~m} / \mathrm{s}$, hence

$$
\frac{\partial \Omega / \partial r H}{\alpha} \simeq 100 \frac{H}{\delta r}
$$

where $H$ denotes the thickness of the convection zone and $\delta r<H$ that of the shear layer, $\delta r=\partial r / \log \Omega$. The quantity (14), therefore, of order $10^{3}$, represents the resulting ratio of the magnetic field components, $\left|B_{\phi}\right| / B_{\mathrm{r}} \mid$. Without circulation the butterfly diagram of course becomes "wrong", with a poleward migration of the toroidal magnetic belts. One can also understand that the counterclockwise flow (poleward at the surface) rapidly destroys the dynamo, in this case already for an amplitude of $20 \mathrm{~m} / \mathrm{s}$. With a value of $5 \times 10^{12} \mathrm{~cm}^{2} / \mathrm{s}$ for the eddy magnetic diffusivity, we find

$$
R m=14 \frac{u^{\mathrm{m}}}{10 \mathrm{~m} / \mathrm{s}},
$$

for the magnetic Reynolds number $R m=u^{\mathrm{m}} R / \eta_{\mathrm{T}} \cdot u^{\mathrm{m}}$ denotes the maximum value of the latitudinal drift close to the bottom of the convection $z^{2} e^{2}$, the circulation is counterclockwise (in the first quadrant) for positive $u^{\mathrm{m}}$.

\footnotetext{
${ }^{2}$ Not to be confused with $u_{0}$, which has the opposite sign for the same flow pattern.
} 

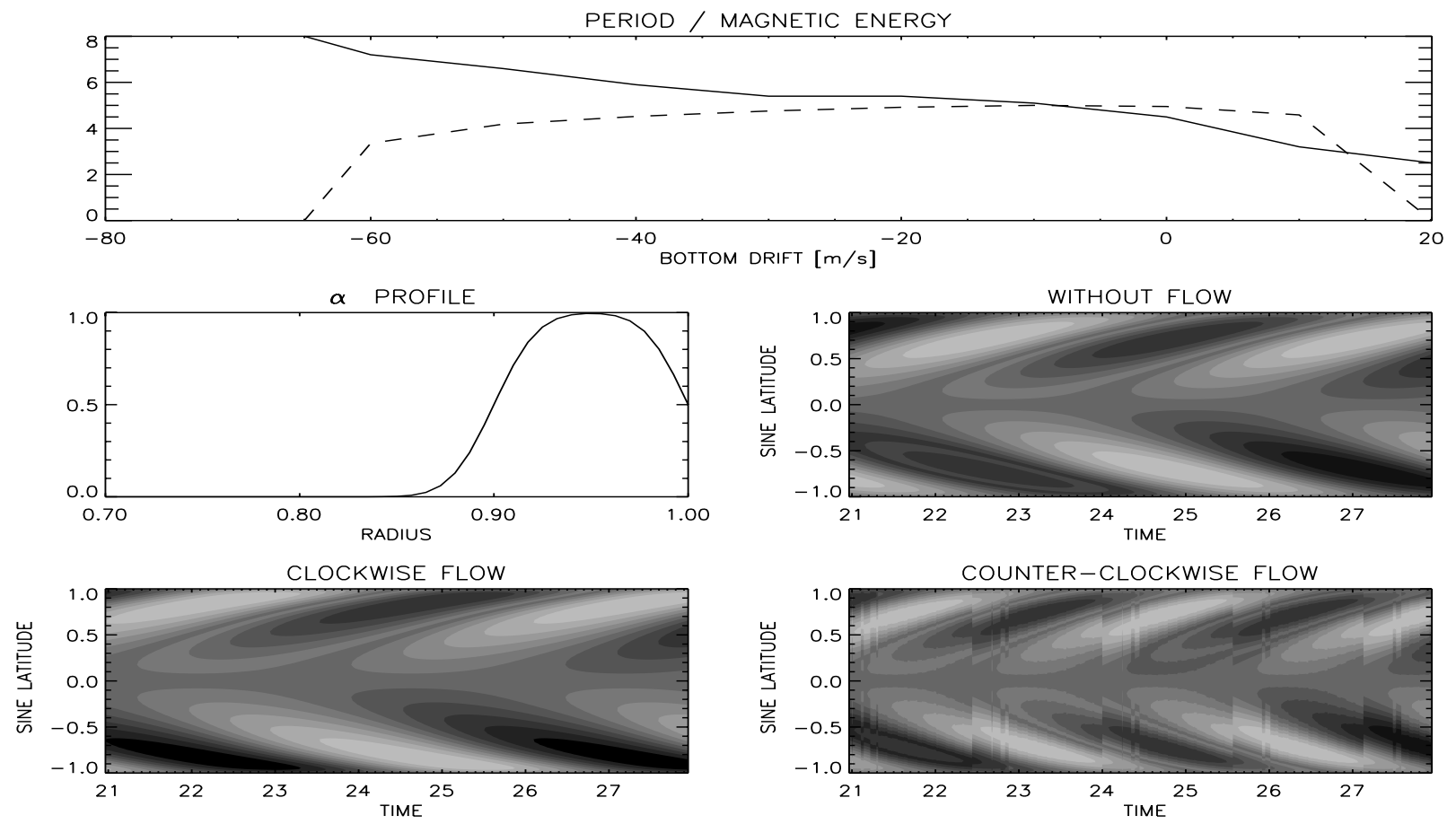

Fig. 4. Operation of a Steenbeck-Krause dynamo with positive alpha-effect (in northern hemisphere) with and without meridional circulation. Eddy diffusivity is $5 \times 10^{12} \mathrm{~cm}^{2} / \mathrm{s}$. Positive bottom drift means counterclockwise flow (equatorward at the bottom of the convection zone, poleward at the surface). Top: the cycle period (solid, in years) and the magnetic energy (dashed) vs. the latitudinal drift at the bottom of the convection zone. Note the decay of the dynamo already for $10 \ldots 20 \mathrm{~m} / \mathrm{s}$ (equatorward at the bottom). The cycle times are basically too short. Middle: $\alpha$-profile in radius and butterfly diagram of the circulation-free dynamo. The maximal toroidal field is plotted vs. the time in years. Bottom: Butterfly diagrams for clockwise and counterclockwise circulations with an amplitude of $10 \mathrm{~m} / \mathrm{s}$ each.

The latter forms a poleward flow at the surface in agreement with observations. For too strong meridional circulation $\left(u^{\mathrm{m}} \geq 20 \mathrm{~m} / \mathrm{s}\right)$ dynamo action ceases. The same happens for $u^{\mathrm{m}} \approx-60 \mathrm{~m} / \mathrm{s}$ if the flow velocity at the bottom is aligned with the dynamo wave vector. As a consequence of the large value of $\eta_{\mathrm{T}}$, the cycle times are shorter than the solar cycle time for all models, a problem that cannot be solved by the meridional flow.

\section{Dynamos with small eddy magnetic diffusivity}

\subsection{A kinematic model}

In order to change the situation fundamentally, the meridional flow must be fast enough, or, more exactly speaking, its magnetic Reynolds number must be large enough. This is only possible with low magnetic eddy diffusivity. We therefore assume a value of $10^{11} \mathrm{~cm}^{2} / \mathrm{s}$ in the convection zone proper, which is estimated from the observed decay times of sunspots, resulting in values of 100-1000 for the magnetic Reynolds number (see also Brandenburg 1993). In their papers, Dikpati \& Choudhuri (1995) and Choudhuri et al. (1995) apply Reynolds numbers of order 500 while Dikpati \& Charbonneau (1999) even take $R m=1400$. Indeed, their dynamos are far beyond the critical values of order of $R m \simeq 10$ of the (circulationfree) $\alpha \Omega$-dynamo. Their dynamos exhibit the same period reduction for increasing $u^{\mathrm{m}}$ as shown in Fig. 4 but the butterfly diagram shows the opposite type of field migration. It seems as if quite another branch of dynamos would exist for $R m \gg 10$. We shall see that this indeed proves to be true.

In Fig. 5 the results from a model with global quenching of the $\alpha$-effect,

$$
q(\boldsymbol{B})=\frac{1}{1+\int \boldsymbol{B}^{2} \mathrm{~d} V},
$$

i.e. quenching with the total magnetic field energy, are presented. This type of $\alpha$ quenching brings the $\alpha$ effect to its critical value and the solution finally found is the marginally stable solution of the linear problem. We may therefore regard dynamos with this type of $\alpha$ quenching as linear. The model is dominated by the $\Omega$ effect and provides a ratio of $\simeq 0.15$ between the amplitudes of the toroidal and the poloidal fields. Despite the positive $\alpha$-effect, the butterfly diagram is solar-type and the cycle time is approximately 20 years. The parameters of this model are close to those observed. Moreover, the phase relation between the toroidal and the radial field components, presented in the second panel of this figure, is negative as observed (Stix 1976). $\alpha \Omega$ dynamos in super-rotating convection zones without meridional flow are known to produce azimuthal and radial field components with the same sign. The circulation dominated dynamo could therefore solve a serious problem of solar 

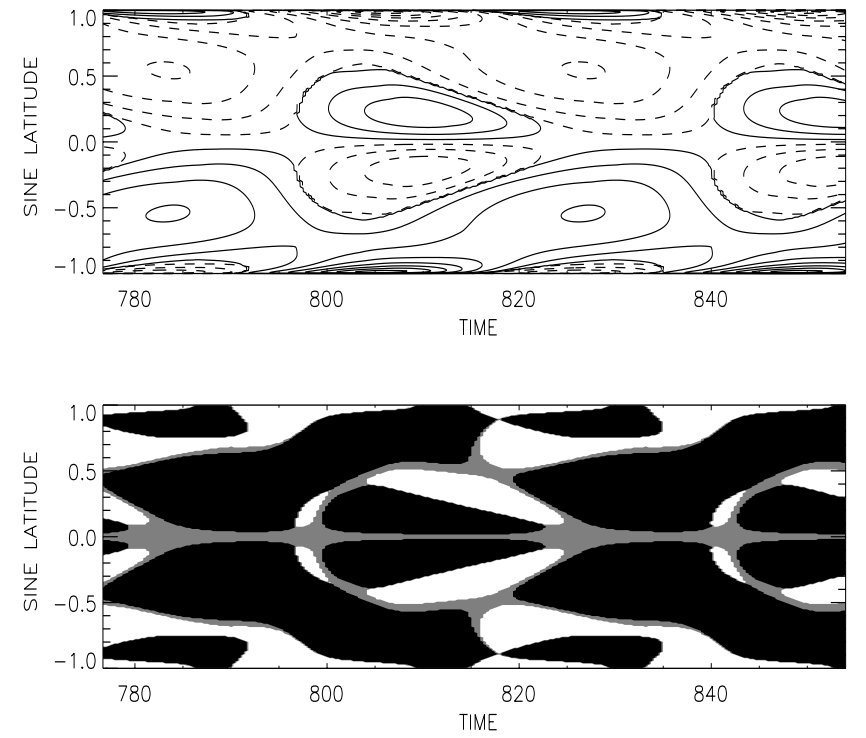

Fig. 5. A linear dynamo: Butterfly diagram (top) and field phase relation (bottom) of a dynamo with positive $\alpha$-effect $\left(\alpha=30 \mathrm{~cm} / \mathrm{s}, \eta_{\mathrm{T}}=10^{11} \mathrm{~cm}^{2} / \mathrm{s}\right)$ and a bottom flow of $1.2 \mathrm{~m} / \mathrm{s}$. Black color in the lower panel means negative $B_{\mathrm{r}} B_{\phi}$.

Table 2. Cycle periods in years of solar-type-butterfly dynamos (bold) with positive $\alpha$-effect $(=3 \mathrm{~m} / \mathrm{s})$ and meridional flow of $u^{\mathrm{m}}$ at the bottom of the convection zone, the eddy diffusivity given in the headline. Signature "_" indicates poleward migration, "dec" means decaying fields.

\begin{tabular}{|r|ccc|}
\hline$u^{\mathrm{m}}[\mathrm{m} / \mathrm{s}]$ & $1 \times 10^{11}$ & $2 \times 10^{11}$ & $5 \times 10^{11}$ \\
\hline 0.24 & -120 & -70 & -36 \\
0.73 & $\mathbf{6 0}$ & -45 & -24 \\
1.22 & $\mathbf{4 5}$ & $\mathbf{4 0}$ & $\mathrm{dec}$ \\
1.71 & $\mathbf{3 1}$ & $\mathbf{2 6}$ & $\mathrm{dec}$ \\
2.69 & $\mathbf{2 0}$ & $\mathbf{2 0}$ & $\mathrm{dec}$ \\
\hline
\end{tabular}

dynamo theory by producing the field components of opposite signs.

\subsection{Nonlinear models}

We have computed a number of models with a standard local $\alpha$-quenching,

$q(\boldsymbol{B})=\frac{1}{1+\boldsymbol{B}^{2}}$,

as the only nonlinearity. All models work with positive (negative) $\alpha$-effect in the northern (southern) hemisphere. The results are summarized in Tables 2 and 3 as well as in Fig. 6.

In Table 2 the (half-)cycle periods are given for dynamos with low eddy diffusivity and various flow amplitudes. Bold numbers indicate those dynamos with a solartype butterfly diagram. We only find them for fast flow
Table 3. The same as in Table 2 but for $\alpha=15 \mathrm{~m} / \mathrm{s}$.

\begin{tabular}{|r|ccl|}
\hline & \multicolumn{3}{|c|}{$\eta_{\mathrm{T}}\left[\mathrm{cm}^{2} / \mathrm{s}\right]$} \\
$u^{\mathrm{m}}[\mathrm{m} / \mathrm{s}]$ & $1 \times 10^{11}$ & $2 \times 10^{11}$ & $5 \times 10^{11}$ \\
\hline 2.69 & $\mathbf{2 4}$ & $\mathbf{2 3}$ & $\mathbf{1 4}$ \\
\hline
\end{tabular}

and low diffusivity. Slow flow and higher diffusivity yield poleward migration of the activity belts. Note also that the cycle period for slow flow runs proportional to $1 / \eta_{\mathrm{T}}$ but not for fast flow. For fast flow the $\alpha$-effect also has a minor influence; only the flow amplitude fixes the cycle time (see Table 3 ).

In Fig. 6 four models are presented for the same turbulence electromotive force but for various amplitudes of the meridional circulation proceeding equatorward at the bottom of the convection zone. The first model has zero flow amplitude. Its butterfly diagram of course has the wrong shape as it works with positive $\alpha . B_{\mathrm{r}} B_{\phi}$ is positive, i.e. also wrong. The latter is changed to negative sign for a meridional flow of $3 \mathrm{~m} / \mathrm{s}$, but the butterfly diagram is still wrong. It starts to change its overall shape at $0.7 \mathrm{~m} / \mathrm{s}$, where the migration of activity belts stops. A meridional flow of $1.2 \mathrm{~m} / \mathrm{s}$ produces a solar-type butterfly diagram with the same $\alpha$-effect and turbulent diffusivity as before. This model also exhibits the observed negative sign of $B_{\mathrm{r}} B_{\phi}$.

\section{Models with solar-type rotation law}

The observed rotation pattern of the solar convection zone has been adopted in the representation of Dikpati \& Charbonneau (1999). It is characterized by the existence of a steep sub-rotation profile in the polar region (cf. Fig. 7). For magnetic fields with dipolar geometry a strong toroidal field always will be induced at polar latitudes unless the eddy diffusivity is increased there. Strong polar activity is not observed, however, on the Sun. All recent dynamo models with the observed rotation law are faced with this problem, even when the $\alpha$-effect has been strongly reduced in the polar region by the relation $\alpha \propto \sin ^{2} \theta \cos \theta$, as we also did.

We study two different radial profiles of the $\alpha$-effect. In the first model, which corresponds to Eq. (6), the $\alpha$ effect exists only in the outer $30 \%$ of the convection zone. The result, shown in Fig. 8, is surprising: there is an oscillation without any drift in latitude. Obviously, the dynamo is driven by the latitudinal shear in the rotation law (Köhler 1973). If almost the whole convection zone forms a positive $\alpha$-effect, as in the second model, the results are very similar. The resulting values of $B_{\mathrm{r}} \cdot B_{\phi}$ are given in Fig. 9, the observed negative sign does not dominate.

We return to the first model and vary the strength of the $\alpha$-effect as well as the amplitude of the meridional flow. Figure 10 shows a series of models with fixed $\alpha_{0}$ and increasing amplitude of the meridional flow. There is 

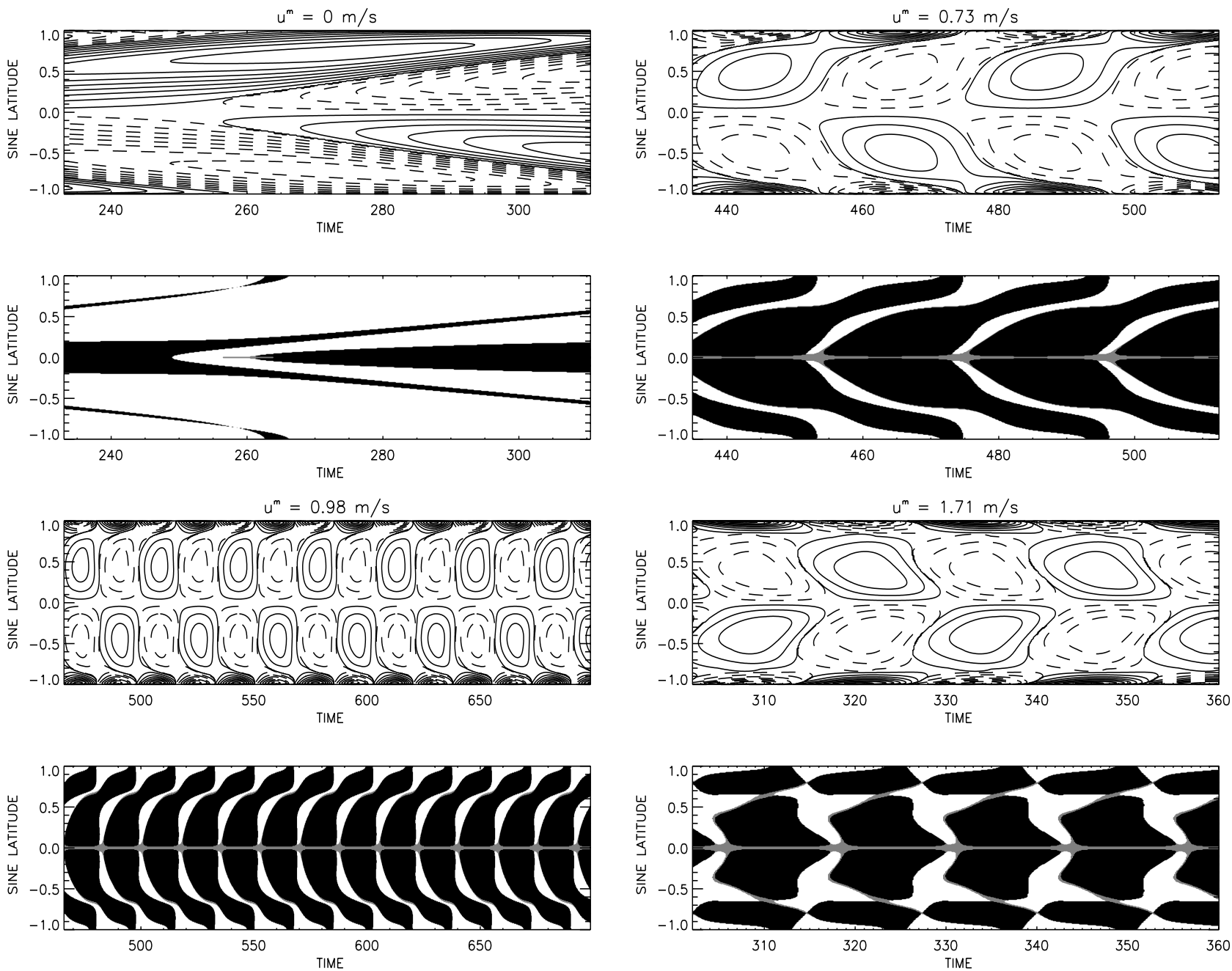

Fig. 6. Dynamo models with positive $\alpha$-effect $(3 \mathrm{~m} / \mathrm{s})$, with eddy diffusivity of $10^{11} \mathrm{~cm}^{2} / \mathrm{s}$ and various bottom values of the meridional flow. Each model is represented by its butterfly diagram (top) and the phase relation of the radial and the toroidal magnetic field components close to the surface. Black color means negative $B_{\mathrm{r}} B_{\phi}$.

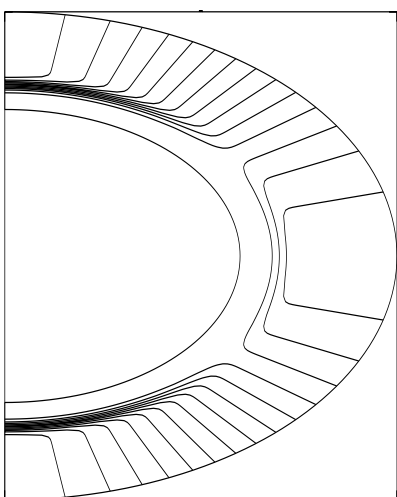

Fig. 7. Isorotation lines used in the models after Dikpati \& Charbonneau (1999).

already a distinct modification of the dynamo for a drift amplitude of only $18 \mathrm{~cm} / \mathrm{s}$. The toroidal field belts are produced in the northern region near the surface. The drift is poleward and indeed the butterfly diagram follows the circulation. This model excellently demonstrates the power
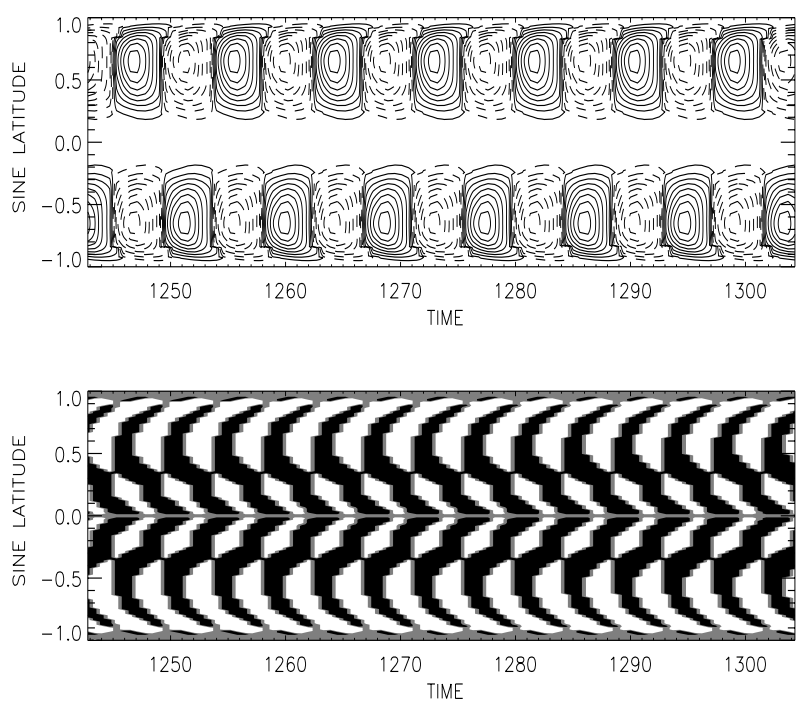

Fig. 8. Butterfly diagram (top) and $B_{\mathrm{r}} \cdot B_{\phi}$-relation (bottom) for dynamo models with the solar rotation law, positive $\alpha$ effect, with eddy diffusivity of $10^{11} \mathrm{~cm}^{2} / \mathrm{s}$ and with thin outer $\alpha$-layer, $\alpha=40 \mathrm{~cm} / \mathrm{s}$, and no meridional flow. 

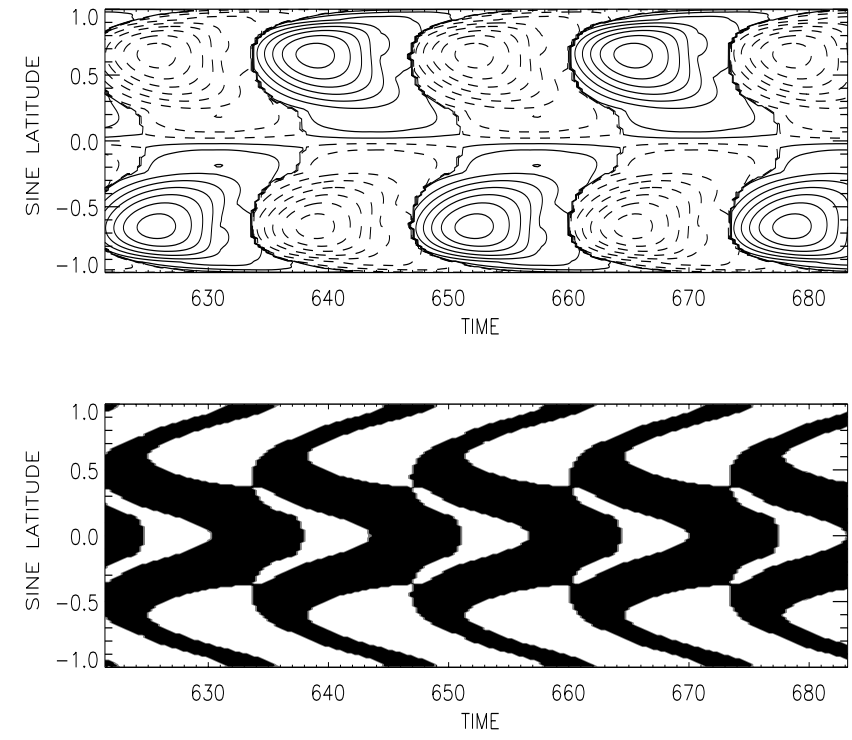

Fig. 9. Butterfly diagram (top) and $B_{\mathrm{r}} \cdot B_{\phi}$-relation (bottom) for dynamo models with the solar rotation law, positive $\alpha$ effect, with eddy diffusivity of $10^{11} \mathrm{~cm}^{2} / \mathrm{s}$ and with thick outer $\alpha$-layer, $\alpha=14 \mathrm{~cm} / \mathrm{s}$, and $u_{0}=0$.

of the meridional flow for models with low eddy diffusivity. However, the toroidal field belts of the dynamo with slow flow are much too far from the equator. Strong toroidal magnetic belts only exist in the polar region and they migrate poleward. The observed equatorward migrating activity region is completely missing. Moreover, the $B_{\mathrm{r}} \cdot B_{\phi}$ are positive almost everywhere, in contrast to the observations. All our circulation-free dynamo models or dynamo models with slow flow, with positive $\alpha$-effect and the rotation law "observed" in the convection zone lead to the same unrealistic results.

Increasing the drift amplitude to $0.88 \mathrm{~m}$ (bottom value) destroys the oscillating dynamo (cf. Fig. 10). The interaction of differential rotation, circulation and $\alpha$-effect does not allow the formation of dynamo waves. Only stationary solutions exist.

However, for $1.23 \mathrm{~m} / \mathrm{s}$ a new branch of solution is already reached with a rather realistic butterfly diagram. Also, the $B_{\mathrm{r}} \cdot B_{\phi}$-diagram shows the desired dominance of negative values. Still, the maximal toroidal fields are concentrated in the polar regions but a clear circulationinduced equatorward migration of the fields can be noted. This type of solution is closest in its character to that of solar activity. Unfortunately, it is not robust. For faster meridional flow the dynamo rapidly ceases to oscillate.

The butterfly diagrams in Fig. 10 together with a number of similar series for different values of $\alpha_{0}$ show a whole "zoo" of solutions, summarized in Fig. 11. With the solar radius $R=7 \times 10^{10} \mathrm{~cm}$ a value of $10^{11} \mathrm{~cm}^{2} / \mathrm{s}$ for the magnetic diffusivity coefficient, and a value of $1 \mathrm{~m} / \mathrm{s}$ for $\alpha_{0}$, we get

$C_{\Omega}=\frac{\partial \Omega / \partial r R^{3}}{\eta_{\mathrm{T}}}=5 \times 10^{4} \quad$ and $\quad C_{\alpha}=\frac{\alpha_{0} R}{\eta_{\mathrm{T}}}=70$

for the magnetic Reynolds numbers associated with differential rotation and $\alpha$ effect, respectively. A value of $1 \mathrm{~m} / \mathrm{s}$ for the meridional flow gives a value of 70 for the magnetic Reynolds number $\mathrm{Rm}$.

Above the critical value of $\alpha_{0}$, the field belts move poleward for slow and equatorward for fast flow. For intermediate flow speeds, the critical value of $\alpha_{0}$ is substantially raised and above it a stationary mode is found. For large flow speeds, the stationary mode is the first to be found above the critical value of $\alpha_{0}$, but then only in a narrow interval. Without any meridional motion at all the magnetic field oscillates but does not show a latitudinal drift. For sufficiently large values of $\alpha_{0}$ the interval of flow speeds for which solar-type butterfly diagrams are found is considerably larger than in the weakly supercritical case shown in Fig. 10.

\section{Conclusions}

For sufficiently small eddy magnetic diffusivity we have shown the meridional flow as very powerful to change the properties of $\alpha^{2} \Omega$-dynamos working in the convection zone rather than in the solar tachocline. For positive but uniform $\partial \Omega / \partial r$ the results are very promising: the migration of the toroidal magnetic activity belts is strongly correlated with the amplitude of the circulation. If the circulation is equatorward at the bottom of the convection zone and its amplitude is sufficient then it can indeed turn a poleward drift into an equatorward drift (see Fig. 6). The resulting cycle times are always between 10 and 100 years (see Tables 2 and 3). Another striking property of the circulation-dominated models is that they produce the observed opposite signs of the magnetic field components $\bar{B}_{\mathrm{r}}$ and $\bar{B}_{\phi}$.

A much more complicated situation arises if the real internal rotation law from helioseismology is applied. The large negative slope $\partial \Omega / \partial r$ in the polar regions unavoidably produces strong toroidal field belts at high latitudes. For these models stationary solutions are found much more frequently than those with cyclic behavior. An equatorward migration of the toroidal field belts (ca. $1 \mathrm{~m} / \mathrm{s}$ at the bottom of the convective zone, see Fig. 10) is only achieved in a very narrow range of flow amplitudes. That solution shows the correct cycle time and also the negativity of $\bar{B}_{\mathrm{r}} \cdot \bar{B}_{\phi}$ but nevertheless only exists for a very special flow strength.

Acknowledgements. M. K. is grateful for the kind support by the Deutsche Forschungsgemeinschaft. 

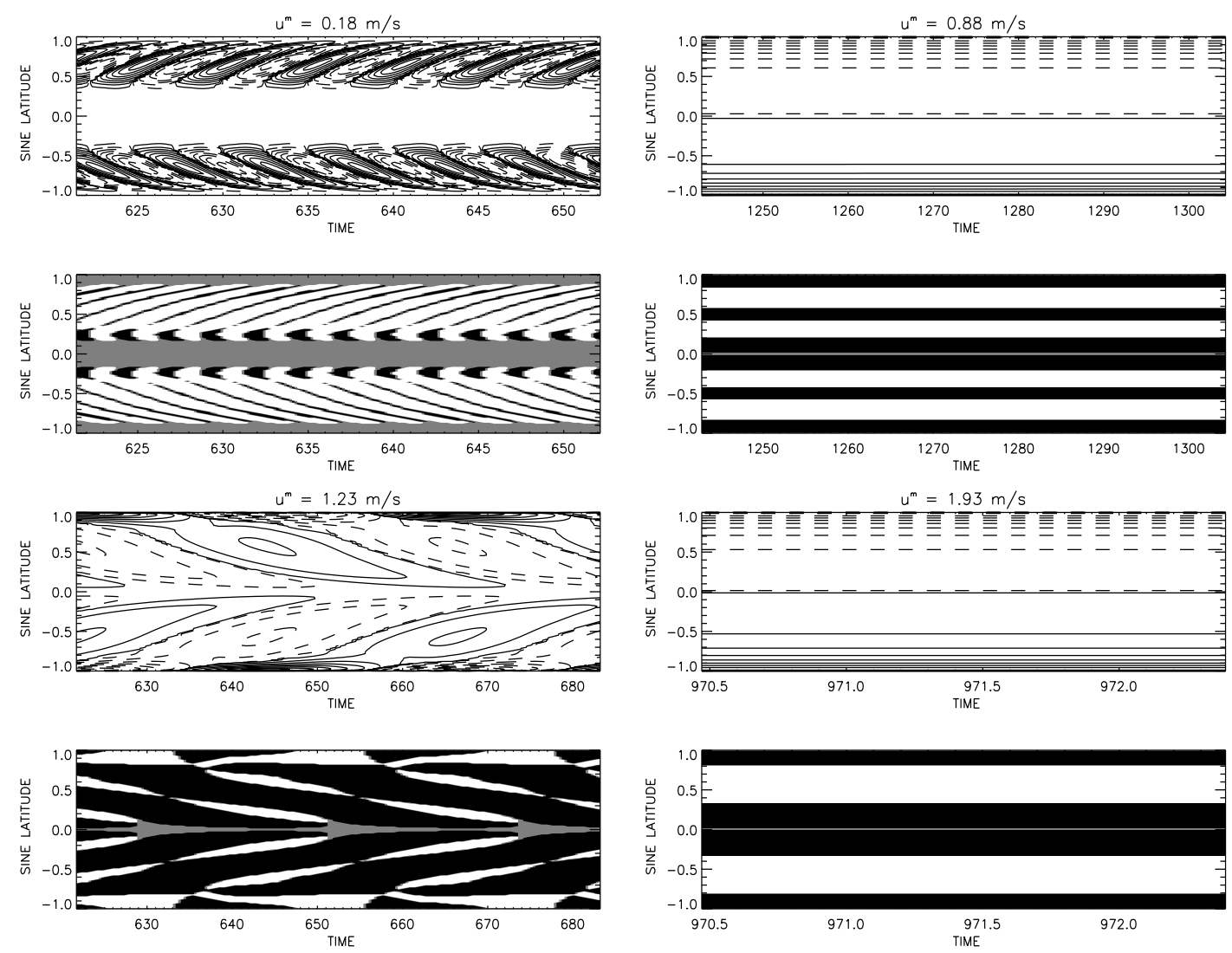

Fig. 10. Dynamo models with the solar rotation law, positive $\alpha$-effect $(40 \mathrm{~cm} / \mathrm{s})$, with eddy diffusivity of $10^{11} \mathrm{~cm}^{2} / \mathrm{s}$ and various bottom values of the meridional flow. Each model is represented by its butterfly diagram (top) and the phase relation of the radial and the toroidal magnetic field components close to the surface. Black color means negative $B_{\mathrm{r}} \cdot B_{\phi}$.

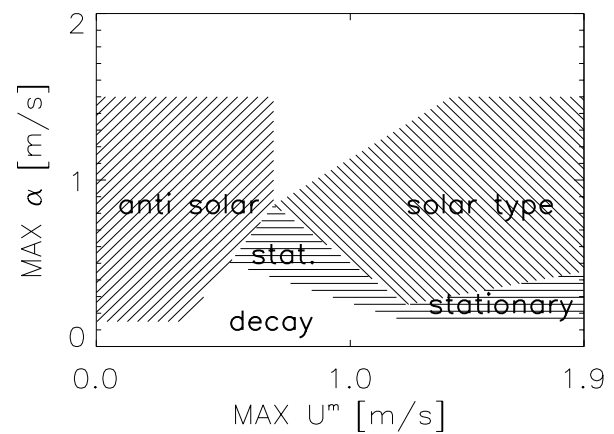

Fig. 11. The different types of solution found for varying strengths of the $\alpha$-effect and the flow speed. The terms solar type and anti solar refer to equatorward and poleward drifting field belts, respectively, while stationary refers to a stationary field. The magnetic diffusivity always has a value of $10^{11} \mathrm{~cm}^{2} / \mathrm{s}$.

\section{References}

Abramenko, V. I., Wang, T., \& Yurchishin, V. B. 1996, Solar Phys., 168, 75

Bao, S., \& Zhang, H. 1998, ApJ, 496, L43

Brandenburg, A. 1993, Simulation the solar dynamo, in IAU Symp. 273, The cosmic dynamo, ed. F. Krause, K.-H. Rädler, \& G. Rüdiger (Kluwer, Dordrecht), 111

Brandenburg, A., \& Schmitt, D. 1998, A\&A, 338, L55

Brandenburg, A. 1999, Helicity in large-scale dynamo simulations, in Magnetic helicity in space and laboratory plasmas, ed. M. R. Brown, R. C. Canfield, \& A. A. Pevtsov (American Geophysical Union, Washington), 65
Choudhuri, A. R., Schüssler, M., \& Dikpati, M. 1995, A\&A, 303, L29

Dikpati, M., \& Choudhuri, A. R. 1995, Solar Phys., 161, 9

Dikpati, M., \& Charbonneau, P. 1999, ApJ, 518, 508

Durney, B. R. 1995, Solar Phys., 160, 213

Durney, B. R. 1996, Solar Phys., 166, 231

Hale, G. E. 1927, Nature, 119, 708

Kitchatinov, L. L., \& Rüdiger, G. 1999, A\&A, 344, 911

Köhler, H. 1973, A\&A, 25, 467

Küker, M., Arlt, R., \& Rüdiger, G. 1999, A\&A, 343, 977

Low, B. C. 1996, Solar Phys., 167, 217

Moss, D. 1999, MNRAS, 306, 300

Moss, D., \& Brooke, J. 2000, MNRAS, 315, 521

Parker, E. N. 1987, Solar Phys., 110, 11

Pevtsov, A. A., Canfield, R. C., \& Metcalf, T. R. 1995, ApJ, 440, L109

Rädler, K.-H., \& Bräuer, H.-J. 1987, Astron. Nachr., 308, 101

Roberts, P. H., \& Stix, M. 1972, A\&A, 18, 453

Rüdiger, G., \& Brandenburg, A. 1995, A\&A, 296, 557

Rüdiger, G., \& Arlt, R. 2000, The physics of the solar cycle, in Advances in nonlinear dynamos, The Fluid Mechanics of Astrophysics and Geophysics, ed. A. Ferriz Mas, \& Núñez Jimenéz, vol. 8

Rüdiger, G., Pipin, V. V., \& Belvedere, G. 2000, Solar Phys., submitted

Schlichenmaier, R., \& Stix, M. 1995, A\&A, 302, 264

Seehafer, N. 1990, Solar Phys., 125, 219

Steenbeck, M., \& Krause, F. 1969, Astron. Nachr., 291, 49

Stix, M. 1973, A\&A, 24, 275

Stix, M. 1976, A\&A, 47, 243 